\title{
Distributed Feedback Effects in Active Semiconductor Photonic Crystal Waveguides
}

\author{
Chen, Yaohui; Mørk, Jesper
}

Published in:

Advanced Photonics Congress

Publication date:

2012

Document Version

Publisher's PDF, also known as Version of record

Link back to DTU Orbit

Citation (APA):

Chen, Y., \& Mørk, J. (2012). Distributed Feedback Effects in Active Semiconductor Photonic Crystal Waveguides. In Advanced Photonics Congress (pp. ITu2C.4). Optical Society of America.

\section{General rights}

Copyright and moral rights for the publications made accessible in the public portal are retained by the authors and/or other copyright owners and it is a condition of accessing publications that users recognise and abide by the legal requirements associated with these rights.

- Users may download and print one copy of any publication from the public portal for the purpose of private study or research.

- You may not further distribute the material or use it for any profit-making activity or commercial gain

- You may freely distribute the URL identifying the publication in the public portal

If you believe that this document breaches copyright please contact us providing details, and we will remove access to the work immediately and investigate your claim. 


\title{
Distributed Feedback Effects in Active Semiconductor Photonic Crystal Waveguides
}

\author{
Yaohui Chen and Jesper Mørk \\ DTU Fotonik, Department of Photonics Engineering, Technical University of Denmark, \\ Oersteds Plads, Building 343, DK 2800, Kgs. Lyngby, Denmark \\ yach@fotonik.dtu.dk
}

\begin{abstract}
We present a rigorous coupled-wave analysis of slow-light effects in active photonic crystal waveguides. The presence of active material leads to coherent distributed feedback effects that significantly alter the magnitude and phase of output fields.
\end{abstract}

(C) 2012 Optical Society of America

OCIS codes: (250.5980) Semiconductor optical amplifiers, (130.5296) Photonic crystal waveguides.

\section{Introduction}

Photonic crystal $(\mathrm{PhC})$ structures have been proposed as a potential waveguide infrastructure for high-density photonic integrated circuits (PICs). Optical amplification is one of the fundamental functionalities, required for compensating attenuation and coupling losses and thus increasing the number of integrated devices. A major advantage in combining $\mathrm{PhC}$ waveguides and active III-V semiconductors may be the possibility to drastically decrease the component length via enhanced light-matter interaction enabled by slow light (SL) propagation [1]. In particular, ultra-compact amplifiers enabled by SL propagation have been suggested [8,9]. One-dimensional coupled-wave analysis based on a perturbative approach has been widely used to investigate the impact of SL effect (large group index) on optical properties of passive $\mathrm{PhC}$ waveguides, e.g. efficient taper design [2], Kerr nonlinearity [3,4] or disorder-induced scattering [5,6]. For perfectly periodic photonic crystal waveguides neglecting Kerr nonlinearity and disorder, a rigorous set of equations for the amplitudes of forward and backward propagating Bloch waves can be derived [7]. In the passive case, these equations are not coupled. However, the presence of active material leads to a coupling between the forward and backward propagating Bloch waves, an effect that was not previously considered. We show here that such feedback effects may have a very large impact on the properties of SL enhanced optical amplifiers. In particular, the feedback effects lead to a limitation on the maximum gain that can be achieved as well as imposing interference effects even in the case of perfect (zero-reflectivity) input facets.

\section{Theory}

In the weak perturbation limit, we approximate the exact solutions of Maxwell equations as superpositions of monochromatic principal (i.e. TE-like) guided Bloch waves (obtained for the corresponding passive structure) with corresponding forward/backward amplitude $\psi_{ \pm}(z)$ along propagation direction z. For simplicity, we describe the carrier-induced complex susceptibility perturbation, as a product of a complex constant $\chi_{\text {pert }}$ and an active material distribution function $F(r)$, which is homogeneously distributed within the high-index dielectric index material over finite stacks of supercells. In contrast to the heuristically derived coupled mode equations based on plane wave expansion [10,11], we can formulate a set of coupled-mode equations based on Lorentz reciprocity theorem for PhC waveguides:

$$
\begin{array}{r}
\partial_{z} \psi_{+}(z)=\frac{i \omega}{c} n_{g z} \chi_{\text {pert }}\left[\delta(z) \psi_{+}+\kappa^{*}(z) e^{-i 2 \beta z} \psi_{-}\right] \\
\partial_{z} \psi_{-}(z)=-\frac{i \omega}{c} n_{g z} \chi_{\text {pert }}\left[\delta(z) \psi_{-}+\kappa(z) e^{i 2 \beta z} \psi_{+}\right] \\
\delta(z) \equiv \frac{a \int_{S}\left[\varepsilon_{0} F(r)|\mathbf{e}|^{2}\right] d S}{\int_{V}\left[\varepsilon_{0} n_{b}^{2}(r)|\mathbf{e}|^{2}+\mu_{0}|\mathbf{h}|^{2}\right] d V}, \kappa(z) \equiv \frac{a \int_{S}\left[\varepsilon_{0} F(r) \mathbf{e} \cdot \mathbf{e}\right] d S}{\int_{V}\left[\varepsilon_{0} n_{b}^{2}(r)|\mathbf{e}|^{2}+\mu_{0}|\mathbf{h}|^{2}\right] d V}
\end{array}
$$

Here, $n_{g z}$ is the group index along the waveguide direction $z, c$ is the speed of light in vacuum, $\varepsilon_{0}, \mu_{0}$ are the electric and magnetic permittivities of free space, $n_{b}(r)$ is the background refractive index, $a$ is the periodicity, $S$ indicates the transverse plane at position $z$ and $V$ is the volume of a supercell. $[\mathbf{e}, \mathbf{h}]$ as normalized electric and magnetic fields of guided Bloch waves at frequency $\omega$ and propagation constant $\beta . \delta(z)$ and $\kappa(z)$ denote the propagation and backscattering coefficient induced by the perturbation due to the active material. The harmonic term $\exp ( \pm i 2 \beta z)$ arises 


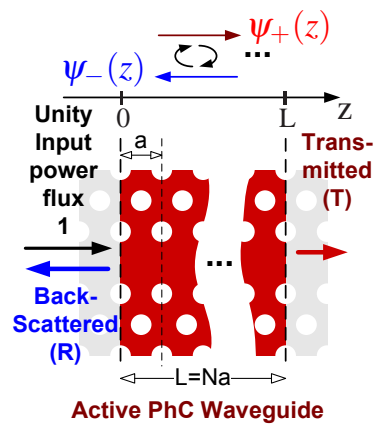

(a)

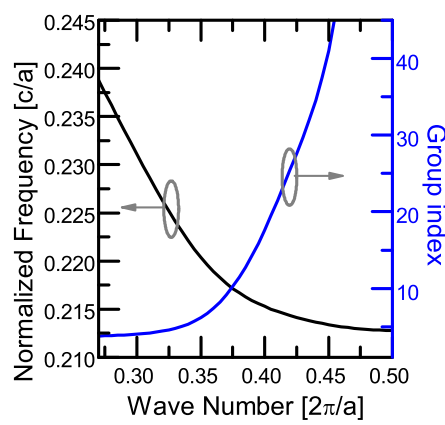

(b)

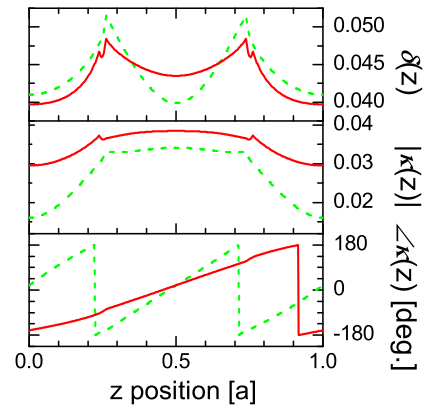

(c)

Fig. 1: (a) Schematic diagram of distributed feedback effects along propagation direction induced by the presence of a gain material.(b) Dispersion properties and calculated group index for defect mode in a 2D W1 PhC waveguide. (c) Parameter examples of a fast light mode (dashed lines $k=0.3[2 \pi / a], n_{g z}=4.027$ ) and a slow light mode (solid lines $k=0.445[2 \pi / a], n_{g z}=37.493$ ). W1 $\mathrm{PhC}$ waveguide parameters: hexagonal lattice, air hole radius $0.26 \mathrm{a}, n_{b}^{2}=11.19$, computational step a/512.

from the phase matching condition between forward and backward Bloch waves. Constrained by Kramers-Kronig relations, we define a simple imaginary refractive index perturbation $n_{i}$ (corresponding to the carrier-induced material gain $g_{0}=-2 n_{i} \omega / c$ ) for the active material. Hereby, the corresponding complex-valued susceptibility perturbation can be described as: $\chi_{\text {pert }}=-n_{i}^{2}+i 2 n_{b} n_{i}$. If neglecting the backscattering and imposing averaging of coefficients within a supercell, i.e., zero-order Fourier coefficient, $\delta^{(0)}=\frac{1}{a} \int_{0}^{a} \delta(z) d z$, Eq. (1-3) are equivalent to the equations previously used for studying SL enhancement of gain/absorption $[8,9]$.

In Fig. 1(b), we show the dispersion properties and calculated group index for TE-like Bloch mode in a passive W1 $\mathrm{PhC}$ waveguide. When the wave number approaches $0.5 \frac{2 \pi}{a}$, both backscattered and forward propagating waves are slow-light enhanced according to Eq. (1)\&(2). Due to different loss mechanisms in practical slow light PhC waveguides $[12,13]$, we limit our discussions to slow light modes with group index up to around 40 (slow light enhancement factor around 10). Fig. 1(c) shows the spatial variations of the coefficients $\delta(z)$ and $\kappa(z)$ for two Bloch modes with different group index. In comparison with the sinusoidally modulated index or gain in the classic coupled mode theory of DFB lasers [10], here $\kappa(z) \exp (i 2 \beta z)$ and its complex conjugate can not be simply approximated as a constant coupling coefficient.

As illustrated in Fig. 1(a), we consider the situation where a CW field is injected at the left facet: $\psi_{+}(0)=1, \psi_{-}(L)=$ 0 , with the input power flux being normalized to 1 . Because our simulations focus on the feedback effects within the active structure rather than the reflection at passive/active interfaces, we enforce continuity boundary conditions. The normalized transmitted $T=\left|\psi_{+}(L)\right|^{2}$ (or backscattered $R=\left|\psi_{-}(0)\right|^{2}$ ) power flux is equal to the device gain defined for traveling-wave (or Reflective) SOAs. The phase of the corresponding outward field amplitudes also reveals the effective optical path length, as altered by coherent feedback. Our approach also permits the inclusion of disorderinduced losses $[5,6]$ and carrier dynamics, which are beyond the scope of this paper.

\section{Numerical Simulations}

In the upper plot of Fig. 2(a), for an active waveguide with length $L=100 a$, we show the transmitted and backscattered power flux of an input CW light exciting a slow light mode $\left(k=0.445[2 \pi / a], n_{g z}=37.493\right)$ as a function of material gain. The dashed line shows the ideal SL enhanced device gain/absorption with no distributed feedback [8,9], the slope of which is proportional to the group index $T \approx \exp \left(2 n_{g z} \delta^{(0)} n_{b} g_{0} L\right)$. In the absorption regime, the increase of the material absorption leads to an increase of backscattered power flux. But the weak backscattered power damped by material loss has negligible feedback effects on the transmitted power. On the other hand, in the regime of positive net gain, the gain material amplifies the light and increases the effective length for wave interaction. As the material gain increases, the transmitted power reaches a maximum value and then steeply decreases. The origin of the drop in device gain is seen to be the increase of the backscattered power. In the bottom plot of Fig. 2(a), the phase of the corresponding outward fields are illustrated. The dashed line shows that the phase variation of the transmitted field with no feedback effects, which is determined by $\approx-\frac{\omega}{c} n_{g z} n_{i}^{2} \delta^{(0)} L$, are negligible. However, by including the distributed feedback effects, the increase of material gain/absorption also contributes to the significant change of phase via amplitude/phase coupling determined by $\kappa(z) \exp (i 2 \beta z)$.

Fig. 2(b) shows an example snapshot of the spatial distribution of the amplitude and phase of forward $\psi_{+}(z)$ and backward $\psi_{-}(z)$ propagating fields, which do not vary monotonically with z. In comparison, the dashed lines indicate 


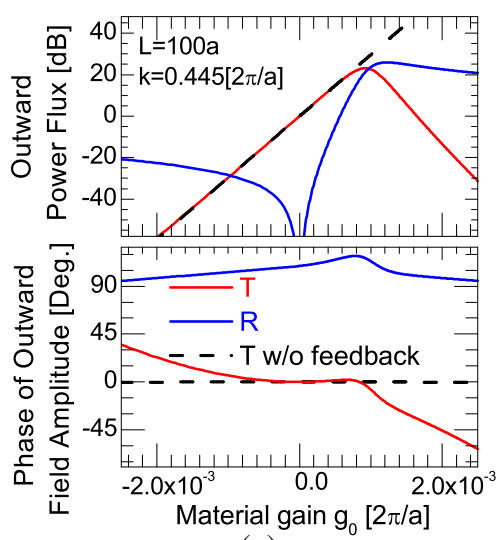

(a)

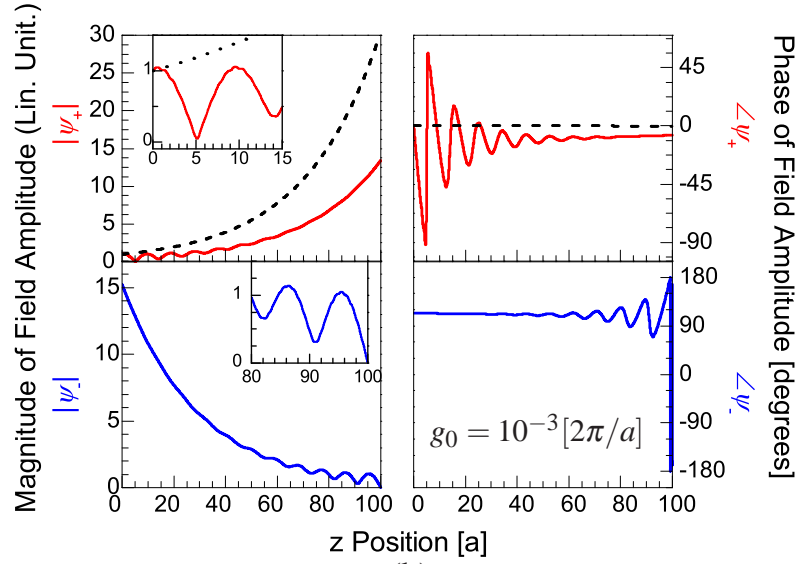

(b)

Fig. 2: (a) Normalized power flux and phase of transmitted and backscattered field amplitudes as a function of material gain $g_{0}$ for a slow light mode. (b) Example of forward and backward field amplitude variations with impact of distributed feedback $g_{0}=10^{-3}[2 \pi / a]$. Dashed lines correspond to results neglecting distributed feedback. Length of the active region is $L=100 a$, $\mathrm{k}=0.445 \times[2 \pi / a], n_{g z}=37.493$.

SL enhanced (traveling wave) amplification and phase change without the inclusion of distributed feedback effects, corresponding to the common exponential variation along the propagation direction which the field being proportional to $\exp \left(i \frac{\omega}{c} n_{g z} \chi_{\text {pert }} \delta^{(0)} z\right)$. For the given material gain, the forward field close to the input end $(\mathrm{z}=0)$ becomes weaker than the backward fields $\left|\psi_{+}(z)\right|<\left|\psi_{-}(z)\right|$. The onset of large phase variations of the forward field $\angle \psi_{+}(z)$ close to the input end indicates a strong coherent coupling between the forward and backward fields. The distance $\Delta z \approx 9 a$ between adjacent magnitude minima of forward field close to the input end $\mathrm{z}=0$ (or backward fields at the output end $\mathrm{z}=\mathrm{L})$ roughly satisfies the phase matching condition $\left(\frac{2 \pi}{a}-2 k\right) \Delta z=2 \pi$.

\section{Conclusions}

We present a Bloch-mode based coupled-wave model for active $\mathrm{PhC}$ waveguides. Our results show that the presence of material gain (an imaginary refractive index perturbation) leads to distributed feedback effects additional to the slow light enhancement of modal gain. These effects severely limit the maximum gain that can be obtained. In addition, when particular phase matching and coupling strength conditions are satisfied, the distributed feedback effects lead to non-negligible coherent interactions in-between counter-propagating Bloch waves.

\section{References}

1. T. Baba, Slow light in photonic crystals, Nature Photonics, 2, 465-473, 2008

2. S.G. Johnson, P. Bienstman, M. A. Skorobogatiy, M. Ibanescu, E. Lidorikis, J. D. Joannopoulos, Adiabatic theorem and continuous coupled-mode theory for efficient taper transitions in photonic crystals, Phys. Rev. E, 66, 066608, 2002

3. J.E.Sipe et al., Effective Field theory for the nonlinear optical properties of photonic crystals, Phys. Rev. E, 69, 016604, 2004

4. N.C. Panoiu et al., Theoretical analysis of pulse dynamics in silicon photonic crystal wire waveguides, IEEE J. Sel. Top. Quantum Electron., 16, 257-266, 2010

5. M. P. Patterson et al., Disorder-induced coherent scattering in Slow-light Photonic crystal waveguides, Phy. Rev. Lett., 102, 253903, 2009

6. S. Mazoyer et al., Disorder-induced multiple scattering in Photonic-Crystal waveguides, Phy. Rev. Lett., 103, 063903, 2009

7. Y. Chen et al., Modelling of active semiconductor photonic crystal waveguides and Robust designs based on topology optimization, in 13th Intl Conf. on Transparent optical Networks (ICTON), 2011, paper We.C4.1

8. E. Mizuta et al., All semiconductor low- $\Delta$ Photonic crystal waveguide for semicondunctor optical amplifier, Japan. J. Appl. Phys. 45, 6116, 2006

9. J. Mørk et al., On the use of slow light for enhancing waveguide properties, Opt. Lett., 35, 2834-2836, 2010

10. H. Kogeinik et al., Coupled-wave theory of distributed feedback lasers, J. Appl. Phys., 43, 2327, 1972

11. S. Olivier et al., Coupled-mode theory and propagation losses in photonic crystal waveguides, Opt. Express, 11, 1490-1496, 2003

12. Y. Tanaka et al., Group velocity dependence of propagation losses in single-line-defect photonic crystal waveguides on GaAs membrane, Electron. Lett., 40, 174-176, 2004

13. L. O'Faolain et al., Loss engineered slow light waveguides, Opt. Express, 18, 27627-27638, 2010 